\title{
Uniqueness of Solutions to a Nonlinear Elliptic Hessian Equation
}

\author{
Siyuan Li \\ School of Mathematics and Applied Statistics, Faculty of Engineering and Information Sciences, University of Wollongong, \\ Wollongong, NSW 2522, Australia \\ Correspondence should be addressed to Siyuan Li; s1296@uowmail.edu.au \\ Received 29 June 2016; Accepted 6 November 2016 \\ Academic Editor: Carlos Conca \\ Copyright (C) 2016 Siyuan Li. This is an open access article distributed under the Creative Commons Attribution License, which \\ permits unrestricted use, distribution, and reproduction in any medium, provided the original work is properly cited.
}

Through an Alexandrov-Fenchel inequality, we establish the general Brunn-Minkowski inequality. Then we obtain the uniqueness of solutions to a nonlinear elliptic Hessian equation on $\mathbb{S}^{n}$.

\section{Introduction}

According to a general Brunn-Minkowski inequality, we obtain a proof of the uniqueness of solutions to the following fully nonlinear elliptic Hessian equation:

$$
\sigma_{k}\left(u_{i j}+u \delta_{i j}\right)=f u^{p-1} \text { on } \mathbb{S}^{n}
$$

where $u$ is the support function of convex bodies, $u_{i j}$ are the second-order covariant derivations of $u$ with respect to any orthonormal frame $\left\{e_{1}, e_{2}, \ldots, e_{n}\right\}$ on $\mathbb{S}^{n}, \delta_{i j}$ is the standard Kronecker symbol, $\mathbb{S}^{n}$ is the unit sphere of $n$-dimension, $f$ is a positive function defined on $\mathbb{S}^{n}, k \in\{1,2, \ldots, n\}, p>1$, and $\sigma_{k}$ is the kth elementary symmetric function defined as follows: for $\lambda=\left(\lambda_{1}, \lambda_{2}, \ldots, \lambda_{n}\right) \in \mathbb{R}^{n}$,

$$
\sigma_{k}(\lambda)=\sum_{1 \leqslant i_{1}<i_{2}<\cdots<i_{k} \leqslant n} \lambda_{i_{1}} \lambda_{i_{2}} \cdots \lambda_{i_{k}} .
$$

The definition can be extended to any symmetric matrix $W \in \mathbb{R}^{n \times n}$ by $\sigma_{k}(W)=\sigma_{k}(\lambda(W))$, where $\lambda(W)=\left(\lambda_{1}(W)\right.$, $\left.\lambda_{2}(W), \ldots, \lambda_{n}(W)\right)$ is the eigenvalue vector of $W$.

Equation (1) arrives from the geometry of convex bodies. A compact convex subset of Euclidean $(n+1)$-space $\mathbb{R}^{n+1}$ with nonempty interiors is called a convex body. An important concept related to a convex body $Q$ is its support function.

Definition 1. Let $M$ (the boundary of a convex body Q) be a smooth, closed, uniformly convex hypersurface enclosing the origin in $\mathbb{R}^{n+1}$. Assume that $M$ is parameterized by its inverse Gauss map $X: \mathbb{S}^{n} \rightarrow M \subset \mathbb{R}^{n+1}$; the support function $u$ of $M$ (or $Q$ ) is defined by

$$
u(x)=\langle x, X(x)\rangle, \quad \forall x \in \mathbb{S}^{n},
$$

where $\langle\cdot, \cdot\rangle$ denotes the standard inner product in $\mathbb{R}^{n+1}$.

$u$ is convex after being extended as a function of homogeneous degree 1 in $\mathbb{R}^{n+1}$. Conversely, any continuous convex function $u$ of homogeneous degree 1 determines a convex body as follows:

$$
Q=\left\{y \in \mathbb{R}^{n+1}: y \cdot x \leqslant u(x), \forall x \in \mathbb{S}^{n}\right\} .
$$

From some basic concepts to support function, Minkowski sum [see Definition 4], and mixed volumes [see Definition 5], Minkowski developed a set of theories related to convex bodies. If $k=n$ and $p=1,(1)$ is the Monge-Ampère equation corresponding to the classical Minkowski problem

$$
\operatorname{det}\left(u_{i j}+u \delta_{i j}\right)=f \quad \text { on } \mathbb{S}^{n}
$$

which has been solved by Nirenberg [1], Pogorelov [2, 3], Cheng and Yau [4], and many others. When $p=1,(1)$ is the classical Christoffel-Minkowski problem:

$$
\sigma_{k}\left(u_{i j}+u \delta_{i j}\right)=f \quad \text { on } \mathbb{S}^{n}
$$


A necessary condition [3] for (6) to have a solution is

$$
\int_{\mathbb{S}^{n}} x_{i} f(x) d s=0, \quad \forall i=1,2, \ldots, n+1,
$$

where $d s$ is the standard area form on $\mathbb{S}^{n}$. Guan et al. [5] obtained that (7) is sufficient for (6) to have an admissible solution [see Definition 6].

Firey [6] generalized the Minkowski sum to $p$-sum [see Definition 4] from $p=1$ to $p \geqslant 1$ in 1962. Later, Lutwak [7] extended the classical surface area measure to the $p$ sum cases. Also in [7], Lutwak first introduced the general Minkowski problem, which is called $L_{p}$-Minkowski problem thereafter. In the smooth category, $L_{p}$-Minkowski problem is equivalent to considering the following Monge-Ampère equation:

$$
\operatorname{det}\left(u_{i j}+u \delta_{i j}\right)=f u^{p-1} \text { on } \mathbb{S}^{n} \text {. }
$$

The uniqueness of $L_{p}$-Minkowski problem for $p>1$ and $p \neq$ $n+1$ (the uniqueness holds up to a dilation if $p=n+1$ ) has been solved in [7]. However, the uniqueness for $p<1$ is difficult and still open. In [8], Jian et al. obtained that, for any $-n-1<p<0$, there exists a positive function $f \in C^{\infty}\left(\mathbb{S}^{n}\right)$ to guarantee that (8) has two different solutions, which means that we need more conditions to consider the uniqueness.

When considering cases $1 \leqslant k<n$, attention is paid to the generalized Christoffel-Minkowski problem. In the smooth category, we need to study the $k$-Hessian equation (1).

For (1), Hu et al. [9] got the existence and uniqueness of solutions to (1) when $1 \leqslant k<n$ and $p>k+1$ under appropriate conditions. However, the uniqueness of (1) when $p<1$ has not been solved well. In this paper, we study the uniqueness of (1) for $p>1$.

Our main result is the following.

Theorem 2. Suppose $u$ is a positive admissible solution of

$$
\sigma_{k}\left(u_{i j}+u \delta_{i j}\right)=f u^{p_{0}} \quad \text { on } \mathbb{S}^{n}
$$

where $1 \leqslant k<n, k \in \mathbb{Z}, p_{0} \in \mathbb{R}^{+} \backslash\{k\}$, and $f$ is a positive function defined on the unit sphere $\mathbb{S}^{n}$ and then the uniqueness holds. If $p_{0}=k$, the uniqueness holds up to a dilation, which means that if $u$ solves (9), then $\left\{a u: \forall a \in \mathbb{R}^{+}\right\}$are the whole solutions of (9).

Remark 3. Here, we rewrite (1) by (9), where $p_{0}=p-1$.

The organization of this paper is as follows. In Section 2, we show some basic concepts and lemmas which have been obtained by Guan et al. in [10]. In Section 3, we prove two useful propositions according to the methods in [11]. In the last section, we prove the main theorem.

\section{Preliminaries}

Definition 4. Given two convex bodies $Q_{1}$ and $Q_{2}$ in $\mathbb{R}^{n+1}$ with respective support functions $u_{1}, u_{2}$, and $\lambda, \mu \geqslant 0(\lambda+\mu>$ $0)$, the Minkowski sum $\lambda Q_{1}+\mu Q_{2} \subset \mathbb{R}^{n+1}$ is defined by the convex body whose support function is $\lambda u_{1}+\mu u_{2}$.
For $p \geqslant 1$, let $Q_{1}$ and $Q_{2}$ be two convex bodies containing the origin in $\mathbb{R}^{n+1}$ in their interiors, and $\lambda, \mu \geqslant 0(\lambda+\mu>0)$. The convex body $\lambda \circ Q_{1}+{ }_{p} \mu \circ Q_{2}$, whose support function is given by $\left(\lambda u_{1}^{p}+\mu u_{2}^{p}\right)^{1 / p}$, is called Firey's $p$-sum of $Q_{1}$ and $Q_{2}$, where " + " means the $p$-summation and "o" means Firey's multiplication.

Definition 5. Let $Q_{1}, Q_{2}, \ldots, Q_{r}$ be convex bodies in $\mathbb{R}^{n+1}$ and the volume of their Minkowski sum

$$
Q=\lambda_{1} Q_{1}+\lambda_{2} Q_{2}+\cdots+\lambda_{r} Q_{r}, \quad \lambda_{i} \geqslant 0
$$

is an $(n+1)$ th degree homogeneous polynomial of the family $\lambda_{1}, \lambda_{2}, \ldots, \lambda_{r}$. Specially, the volume of $Q$ is

$$
\begin{aligned}
& \operatorname{Vol}(Q)=\operatorname{Vol}\left(\lambda_{1} Q_{1}+\lambda_{2} Q_{2}+\cdots+\lambda_{r} Q_{r}\right) \\
& =\sum_{i_{1}, i_{2}, \ldots, i_{n+1}=1}^{r} \lambda_{i_{1}} \lambda_{i_{2}} \cdots \lambda_{i_{n+1}} V\left(Q_{i_{1}}, Q_{i_{2}}, \ldots, Q_{i_{n+1}}\right)
\end{aligned}
$$

where the functions $V$ are symmetric. Then $V\left(Q_{1}, Q_{2}, \ldots\right.$, $\left.Q_{n+1}\right)$ is called the Minkowski mixed volume of $Q_{1}, Q_{2}, \ldots$, $Q_{n+1}$.

Definition 6. For $k \in\{1,2, \ldots, n\}$, let $\Gamma_{k}$ be the convex cone in $\mathbb{R}^{n}$ which is determined by

$$
\Gamma_{k}=\left\{\lambda \in \mathbb{R}^{n}: \sigma_{1}(\lambda)>0, \sigma_{2}(\lambda)>0, \ldots, \sigma_{k}(\lambda)>0\right\} .
$$

A function $u \in C^{2}\left(\mathbb{S}^{n}\right)$ is called $k$-convex if

$$
W(x)=\left\{u_{i j}(x)+u(x) \delta_{i j}\right\} \in \Gamma_{k}, \quad \forall x \in \mathbb{S}^{n}
$$

and $u$ is called an admissible solution to (1) if $u$ is $k$-convex and satisfies (1).

Definition 7. Let $A_{1}, A_{2}, \ldots, A_{m}$ be symmetric real $k \times k$ matrices, $\lambda_{1}, \lambda_{2}, \ldots, \lambda_{m} \in \mathbb{R}$; the determinant of $\lambda_{1} A_{1}+$ $\cdots+\lambda_{m} A_{m}$ is a homogeneous polynomial of degree $k$ in $\lambda_{1}, \lambda_{2}, \ldots, \lambda_{m}$. Namely,

$$
\begin{aligned}
\operatorname{det} & \left(\lambda_{1} A_{1}+\cdots+\lambda_{m} A_{m}\right) \\
= & \sum_{i_{1}, \ldots, i_{k}=1}^{m} \lambda_{i_{1}} \cdots \lambda_{i_{k}} D_{k}\left(A_{i_{1}}, \ldots, A_{i_{k}}\right) .
\end{aligned}
$$

In fact, the coefficient $\lambda_{i_{1}} \cdots \lambda_{i_{k}}$ depends only on $A_{i_{1}}, \ldots, A_{i_{k}}$; then they are uniquely determined. $D_{k}\left(A_{1}, \ldots, A_{k}\right)$ is called the mixed discriminant of $A_{1}, \ldots, A_{k}$.

For later applications, we collect some results here which have been proved in [10].

Lemma 8. Let $u_{1}, u_{2}, \ldots, u_{n+1}$ be the support function of convex bodies $Q_{1}, Q_{2}, \ldots, Q_{n+1}$, respectively. Denoting Minkowski mixed volume $V\left(Q_{1}, Q_{2}, \ldots, Q_{n+1}\right)$ by $V\left(u_{1}, u_{2}, \ldots, u_{n+1}\right)$ and

$$
W_{m}=\left\{\left(u_{m}\right)_{i j}+u_{m} \delta_{i j}\right\}, \quad m=1,2, \ldots, n+1,
$$


then

$$
\begin{aligned}
& V\left(u_{1}, u_{2}, \ldots, u_{n+1}\right) \\
& \quad=\int_{\mathbb{S}^{n}} u_{1} D_{n}\left(W_{2}, W_{3}, \ldots, W_{n+1}\right) d s,
\end{aligned}
$$

where $D_{n}\left(W_{2}, W_{3}, \ldots, W_{n+1}\right)$ is the mixed discriminant [see Definition 7] of $W_{2}, W_{3}, \ldots, W_{n+1}$.

Remark 9. For all $1 \leqslant k \leqslant n$, setting $u_{k+2}=\cdots=u_{n+1}=1$, then

$$
\begin{aligned}
& V\left(u_{1}, \ldots, u_{k+1}, 1, \ldots, 1\right):=V_{k+1}\left(u_{1}, u_{2}, \ldots, u_{k+1}\right) \\
& \quad=\int_{\mathbb{S}^{n}} u_{1} D_{k}\left(W_{2}, W_{3}, \ldots, W_{k+1}\right) d s
\end{aligned}
$$

where $D_{k}\left(W_{2}, W_{3}, \ldots, W_{k+1}\right)$ is the mixed discriminant of $W_{2}, W_{3}, \ldots, W_{k+1}$. Furthermore, if $u_{1}=u_{2}=\cdots=$ $u_{n+1}=u$, denote $V\left(u_{1}, u_{2}, \ldots, u_{n+1}\right):=V(u)$ and $V_{k+1}\left(u_{1}\right.$, $\left.u_{2}, \ldots, u_{k+1}\right):=V_{k+1}(u)$; then

$$
\begin{aligned}
V(u) & =\int_{\mathbb{S}^{n}} u \operatorname{det}\left(u_{i j}+u \delta_{i j}\right) d s, \\
V_{k+1}(u) & =\int_{\mathbb{S}^{n}} u \sigma_{k}\left(u_{i j}+u \delta_{i j}\right) d s .
\end{aligned}
$$

Lemma 10. $V$ is a symmetric multilinear form on $\left(C^{2}\left(\mathbb{S}^{n}\right)\right)^{n+1}$.

Lemma 11. For any function $u \in C^{2}\left(\mathbb{S}^{n}\right), W=\left\{u_{i j}+u \delta_{i j}\right\}$, $1 \leqslant k<n$, we have the Minkowski type integral formula,

$$
\int_{\mathbb{S}^{n}} u \sigma_{k}(W) d s=\int_{\mathbb{S}^{n}} \sigma_{k+1}(W) d s,
$$

where ds is the standard area element on $\mathbb{S}^{n}$.

The following is a form of Alexandrov-Fenchel inequality for positive $k$-convex functions which comes from [10].

Lemma 12 (Alexandrov-Fenchel inequality). If $u_{1}, u_{2}, \ldots, u_{k}$ are $k$-convex, $u_{1}$ is positive, and there exists $l \in\{2,3, \ldots, k\}$ such that $u_{l} \geqslant 0$ on $\mathbb{S}^{n}$, then, for any $v \in C^{2}\left(\mathbb{S}^{n}\right)$,

$$
\begin{aligned}
& V_{k+1}^{2}\left(v, u_{1}, u_{2}, \ldots, u_{k}\right) \\
& \quad \geqslant V_{k+1}\left(u_{1}, u_{1}, u_{2}, \ldots, u_{k}\right) V_{k+1}\left(v, v, u_{2}, \ldots, u_{k}\right),
\end{aligned}
$$

with equality if and only if $v=a u_{1}+\sum_{i=1}^{n+1} a_{i} x_{i}$ for some constants $a, a_{1}, \ldots, a_{n+1}$.

\section{Two Important Propositions}

Now we prove two important propositions. The methods we use are from [11].

Proposition 13. Suppose $u_{0}, u_{1}>0$ are $k$-convex; then

$$
\begin{aligned}
& V_{k+1}^{1 /(k+1)}\left((1-t) u_{0}+t u_{1}\right) \\
& \quad \geqslant(1-t) V_{k+1}^{1 /(k+1)}\left(u_{0}\right)+t V_{k+1}^{1 /(k+1)}\left(u_{1}\right),
\end{aligned}
$$

with equality if and only if $u_{0}=a u_{1}+\sum_{i=1}^{n+1} a_{i} x_{i}$ for some constants $a, a_{1}, \ldots, a_{n+1}$.

Proof. We only need to prove that

$$
F(t)=V_{k+1}^{1 /(k+1)}\left((1-t) u_{0}+t u_{1}\right)
$$

is concave on $[0,1]$. Setting $u_{t}=(1-t) u_{0}+t u_{1}, t \in[0,1]$, we have

$$
F(t)=V_{k+1}^{1 /(k+1)}(\overbrace{u_{t}, u_{t}, \ldots, u_{t}}^{k+1}) .
$$

By the symmetric multilinear property of $V$, it is obvious that

$$
\begin{aligned}
& F^{\prime}(t)=V_{k+1}^{1 /(k+1)-1}(\overbrace{u_{t}, \ldots, u_{t}}^{k+1}) V_{k+1}\left(-u_{0}\right. \\
& +u_{1}, \overbrace{u_{t}, \ldots, u_{t}}^{k}) \text {, } \\
& F^{\prime \prime}(t)=k V_{k+1}^{1 /(k+1)-2}(\overbrace{u_{t}, \ldots, u_{t}}^{k+1}) \\
& \cdot\left[V_{k+1}(\overbrace{u_{t}, \ldots, u_{t}}^{k+1})\right. \\
& \cdot V_{k+1}(-u_{0}+u_{1},-u_{0}+u_{1}, \overbrace{u_{t}, \ldots, u_{t}}^{k-1}) \\
& \left.-V_{k+1}^{2}(-u_{0}+u_{1}, \overbrace{u_{t}, \ldots, u_{t}}^{k})\right] \leqslant 0,
\end{aligned}
$$

where the last inequality uses (20); thus $F$ is a concave function on $[0,1]$. The equality condition is checked easily.

Proposition 14 (general Brunn-Minkowski inequality). Supposing $u_{0}, u_{1}>0$ are $k$-convex, then

$$
\begin{aligned}
& \int_{\mathbb{S}^{n}} u_{1} \sigma_{k}\left(\left(u_{0}\right)_{i j}+u_{0} \delta_{i j}\right) d s \\
& \quad \geqslant V_{k+1}^{1 /(k+1)}\left(u_{1}\right) V_{k+1}^{1-1 /(k+1)}\left(u_{0}\right),
\end{aligned}
$$

with equality if and only if $u_{0}=a u_{1}+\sum_{i=1}^{n+1} a_{i} x_{i}$ for some constants $a, a_{1}, \ldots, a_{n+1}$.

Proof. Setting

$$
\begin{aligned}
F(t)= & V_{k+1}^{1 /(k+1)}\left((1-t) u_{0}+t u_{1}\right) \\
& -(1-t) V_{k+1}^{1 /(k+1)}\left(u_{0}\right)-t V_{k+1}^{1 /(k+1)}\left(u_{1}\right),
\end{aligned}
$$

then $F(0)=F(1)=0$. By $(21), F(t) \geqslant 0$; thus $F^{\prime}(0) \geqslant 0$; namely,

$$
\begin{gathered}
V_{k+1}^{1 /(k+1)-1}\left(u_{0}\right) V_{k+1}(-u_{0}+u_{1}, \overbrace{u_{0}, \ldots, u_{0}}^{k}) \\
+V_{k+1}^{1 /(k+1)}\left(u_{0}\right)-V_{k+1}^{1 /(k+1)}\left(u_{1}\right) \geqslant 0 .
\end{gathered}
$$


Then

$$
\begin{aligned}
& V_{k+1}^{1 /(k+1)-1}\left(u_{0}\right) \int_{\mathbb{S}^{n}}\left(-u_{0}+u_{1}\right) \sigma_{k}\left(\left(u_{0}\right)_{i j}+u_{0} \delta_{i j}\right) d s \\
& \quad+V_{k+1}^{1 /(k+1)}\left(u_{0}\right) \geqslant V_{k+1}^{1 /(k+1)}\left(u_{1}\right) .
\end{aligned}
$$

By (19),

$$
\begin{aligned}
& V_{k+1}^{1 /(k+1)-1}\left(u_{0}\right) \int_{\mathbb{S}^{n}} u_{1} \sigma_{k}\left(\left(u_{0}\right)_{i j}+u_{0} \delta_{i j}\right) d s \\
& \quad \geqslant V_{k+1}^{1 /(k+1)}\left(u_{1}\right)
\end{aligned}
$$

and then

$$
\begin{aligned}
& \int_{\mathbb{S}^{n}} u_{1} \sigma_{k}\left(\left(u_{0}\right)_{i j}+u_{0} \delta_{i j}\right) d s \\
& \quad \geqslant V_{k+1}^{1 /(k+1)}\left(u_{1}\right) V_{k+1}^{1-1 /(k+1)}\left(u_{0}\right) .
\end{aligned}
$$

\section{Proof of Theorem 2}

Now we prove Theorem 2 . The main methods are from $[7,12]$.

Proof. Assuming that (9) has two solutions $u$ and $v$, then we consider the equation in the following three cases.

Case $1\left(p_{0}>k\right)$. Supposing $x_{0}$ is the maximum value point of $G=u / v$, then at $x_{0}$, we have

$$
\begin{aligned}
0 & =\nabla \ln G=\frac{\nabla u}{u}-\frac{\nabla v}{v} \\
0 & \geqslant \nabla^{2} \ln G=\left(\frac{\nabla^{2} u}{u}-\frac{(\nabla u)^{2}}{u^{2}}\right)-\left(\frac{\nabla^{2} v}{v}-\frac{(\nabla v)^{2}}{v^{2}}\right) \\
& =\frac{\nabla^{2} u}{u}-\frac{\nabla^{2} v}{v}
\end{aligned}
$$

that is,

$$
\frac{\nabla^{2} u}{u} \leqslant \frac{\nabla^{2} v}{v}
$$

Hence

$$
\begin{aligned}
f u^{p_{0}}\left(x_{0}\right) & =u^{k}\left(x_{0}\right) \sigma_{k}\left(\frac{u_{i j}}{u}+\delta_{i j}\right)\left(x_{0}\right) \\
& \leqslant u^{k}\left(x_{0}\right) \sigma_{k}\left(\frac{v_{i j}}{v}+\delta_{i j}\right)\left(x_{0}\right) \\
& =\frac{u^{k}\left(x_{0}\right)}{v^{k}\left(x_{0}\right)} f v^{p_{0}}\left(x_{0}\right) ;
\end{aligned}
$$

therefore

$$
u^{p_{0}-k}\left(x_{0}\right) \leqslant v^{p_{0}-k}\left(x_{0}\right) \Longrightarrow G\left(x_{0}\right)=\frac{u\left(x_{0}\right)}{v\left(x_{0}\right)} \leqslant 1
$$

then

$$
\frac{u}{v} \leqslant 1
$$

Similarly, we have $v / u \leqslant 1$. Thus $u \equiv v$.

Case $2\left(0<p_{0}<k\right)$. We have

$$
u^{-p_{0}} \sigma_{k}\left(u_{i j}+u \delta_{i j}\right)=v^{-p_{0}} \sigma_{k}\left(v_{i j}+v \delta_{i j}\right) ;
$$

then

$$
\begin{aligned}
& V_{k+1}(u)=\int_{\mathbb{S}^{n}} u \sigma_{k}\left(u_{i j}+u \delta_{i j}\right) d s \\
& =\int_{\mathbb{S}^{n}}\left(\frac{u}{v}\right)^{p_{0}+1} v \sigma_{k}\left(v_{i j}+v \delta_{i j}\right) d s \\
& \geqslant\left[\int_{\mathbb{S}^{n}} u \sigma_{k}\left(v_{i j}+v \delta_{i j}\right) d s\right]^{p_{0}+1} \\
& \cdot\left[\int_{\mathbb{S}^{n}} v \sigma_{k}\left(v_{i j}+v \delta_{i j}\right) d s\right]^{-p_{0}} \geqslant V_{k+1}^{\left(p_{0}+1\right) /(k+1)}(u) \\
& \cdot V_{k+1}^{\left(k p_{0}+k\right) /(k+1)}(v) V_{k+1}^{-p_{0}}(v)=V_{k+1}^{\left(p_{0}+1\right) /(k+1)}(u) \\
& \cdot V_{k+1}^{1-\left(p_{0}+1\right) /(k+1)}(v),
\end{aligned}
$$

where we have used Hölder inequality in the first inequality and used (26) in the second one. Hence $V_{k+1}(u)=V_{k+1}(v)$, which forces both the equalities to hold. By the equality condition, there exists a constant $a \in \mathbb{R}$ such that $v=a u$. By (9), we know $a=1$. Therefore, $u \equiv v$.

Case $3\left(p_{0}=k\right)$. According to Case 2 , when $p_{0}=k$, we have

$$
\begin{aligned}
& V_{k+1}(u)=\int_{\mathbb{S}^{n}} u \sigma_{k}\left(u_{i j}+u \delta_{i j}\right) d s=\int_{\mathbb{S}^{n}}\left(\frac{u}{v}\right)^{k+1} \\
& \cdot v \sigma_{k}\left(v_{i j}+v \delta_{i j}\right) d s \geqslant\left[\int_{\mathbb{S}^{n}} u \sigma_{k}\left(v_{i j}+v \delta_{i j}\right) d s\right]^{k+1} \\
& \cdot\left[\int_{\mathbb{S}^{n}} v \sigma_{k}\left(v_{i j}+v \delta_{i j}\right) d s\right]^{-k} \geqslant V_{k+1}(u) V_{k+1}^{k}(v) \\
& \cdot V_{k+1}^{-k}(v)=V_{k+1}(u) ;
\end{aligned}
$$

then all the equalities hold. Thus there exists $a \in \mathbb{R}$, such that $v=a u$. Therefore $\left\{a u: \forall a \in \mathbb{R}^{+}\right\}$are the whole solutions of (9).

Now we complete the proof of Theorem 2.

\section{Competing Interests}

The author declares no competing interests.

\section{References}

[1] L. Nirenberg, "The Weyl and Minkowski problems in differential geometry in the large," Communications on Pure and Applied Mathematics, vol. 6, no. 3, pp. 337-394, 1953. 
[2] A. V. Pogorelov, "On existence of a convex surface with a given sum principal radii of curvature," Uspekhi Matematicheskikh Nauk, vol. 8, no. 3, pp. 127-130, 1953 (Russian).

[3] A. V. Pogorelov, The Multidimensional Minkowski Problem, Winston, Washington, DC, USA, 1978.

[4] S. Y. Cheng and S. T. Yau, "On the regularity of the solution of the $n$-dimensional Minkowski problem," Communications on Pure and Applied Mathematics, vol. 29, no. 5, pp. 495-516, 1976.

[5] P. Guan, X.-N. Ma, and F. Zhou, "The Christofel-Minkowski problem. III. Existence and convexity of admissible solutions," Communications on Pure and Applied Mathematics, vol. 59, no. 9, pp. 1352-1376, 2006.

[6] W. J. Firey, "p-means of convex bodies," Mathematica Scandinavica, vol. 10, pp. 17-24, 1962.

[7] E. Lutwak, "The Brunn-Minkowski-Firey theory I: mixed volumes and the Minkowski problem," Journal of Differential Geometry, vol. 38, no. 1, pp. 131-150, 1993.

[8] H. Jian, J. Lu, and X.-J. Wang, "Nonuniqueness of solutions to the $L_{p}$-Minkowski problem," Advances in Mathematics, vol. 281, pp. 845-856, 2015.

[9] C. Q. Hu, X.-N. Ma, and C. Shen, "On the ChristoffelMinkowski problem of Firey's p-sum," Calculus of Variations and Partial Differential Equations, vol. 21, no. 2, pp. 137-155, 2004.

[10] P. F. Guan, X.-N. Ma, N. Trudinger, and X. Zhu, "A form of Alexandrov-Fenchel inequality," Pure and Applied Mathematics Quarterly, vol. 6, no. 4, pp. 999-1012, 2010.

[11] R. Schneider, Convex Bodies: the Brunn-Minkowski Theory, Cambridge University Press, Cambridge, UK, 2013.

[12] K.-S. Chou and X.-J. Wang, "The $L_{p}$-Minkowski problem and the Minkowski problem in centroaffine geometry," Advances in Mathematics, vol. 205, no. 1, pp. 33-83, 2006. 


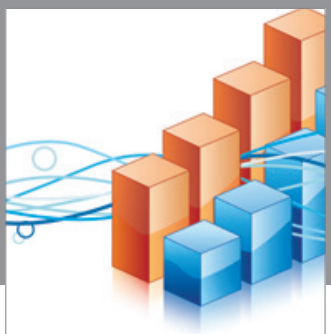

Advances in

Operations Research

vatem alat4

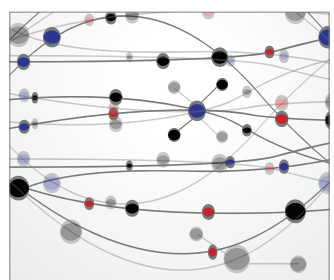

\section{The Scientific} World Journal
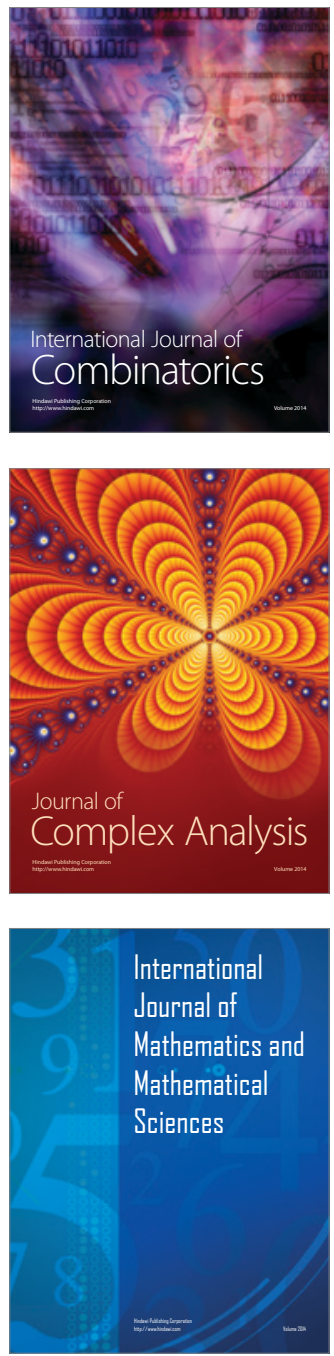
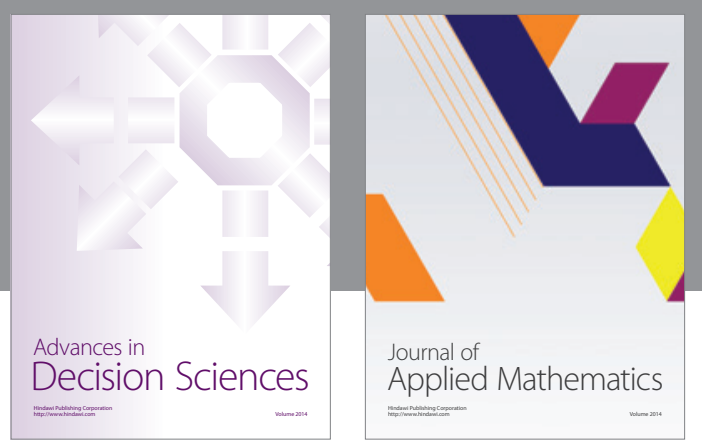

Algebra

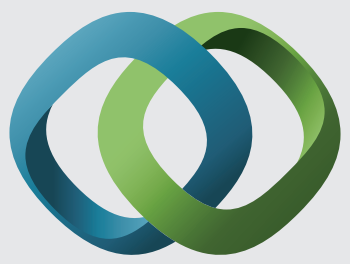

\section{Hindawi}

Submit your manuscripts at

http://www.hindawi.com
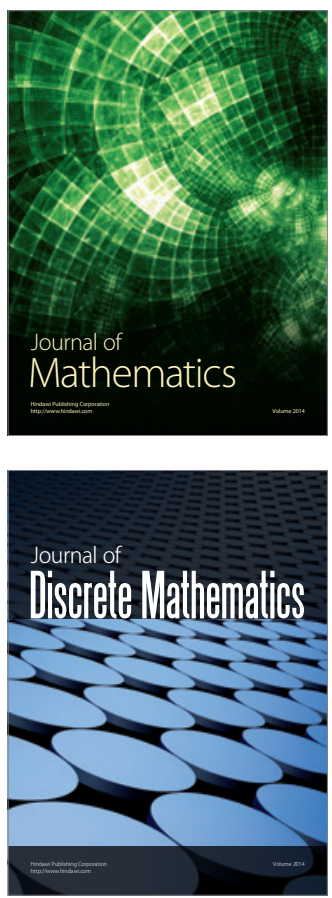

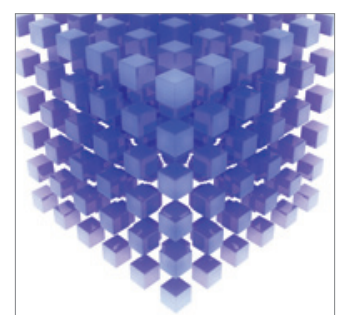

Mathematical Problems in Engineering
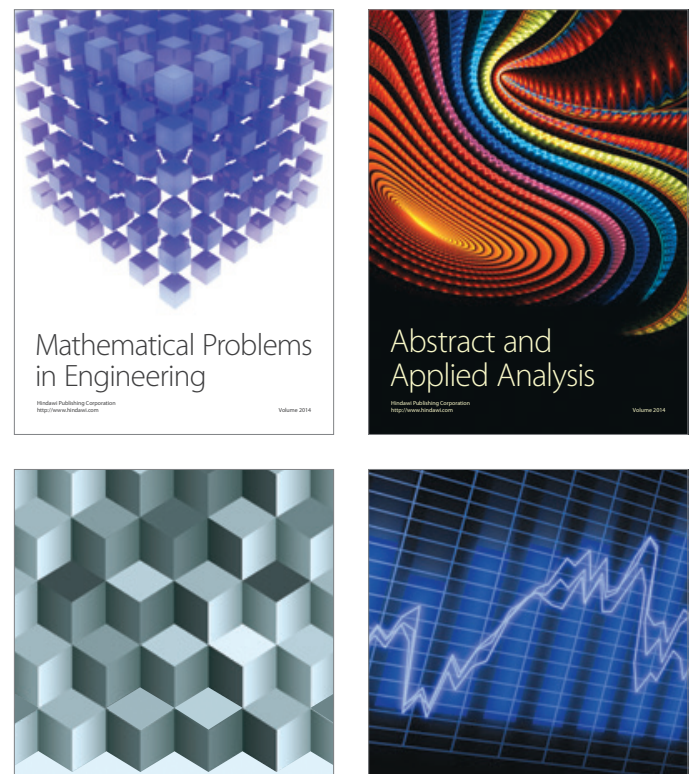

Journal of

Function Spaces

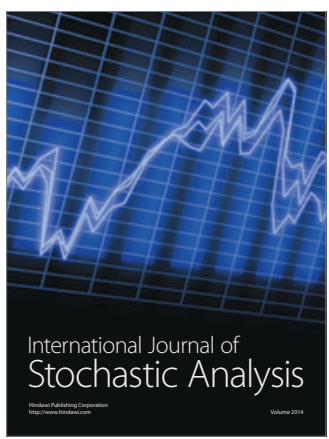

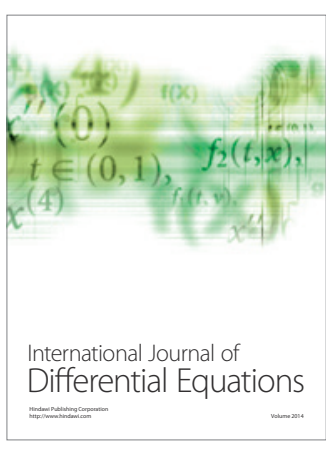
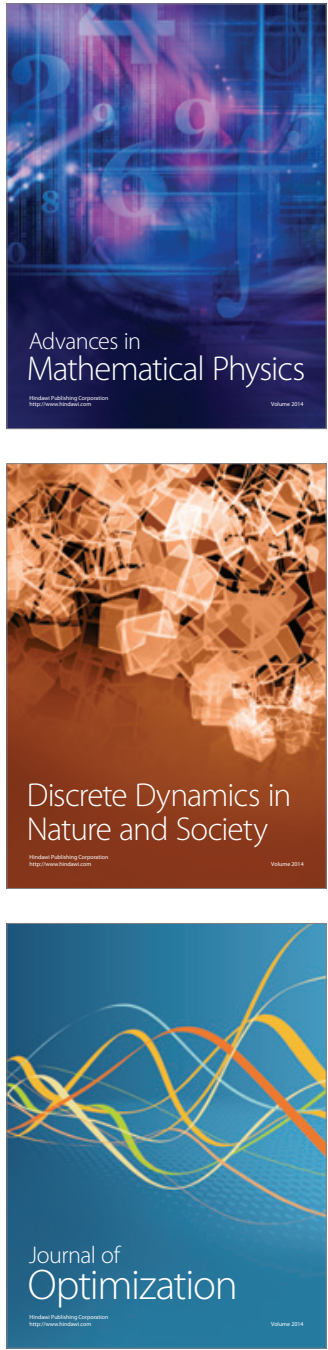